Research Paper

\title{
PD-1 Blockade Overcomes Adaptive Immune Resistance in Treatment with Anchored-GM-CSF Bladder Cancer Cells Vaccine
}

\author{
Xinji Zhang ${ }^{1}$, Xiaojun Shi², Jinlong $\mathrm{Li}^{3}$, Lijun $\mathrm{Mo}^{3}$, Zhiming $\mathrm{Hu}^{3}$, Jimin $\mathrm{Gao}^{4}$, Shihao $\mathrm{Wu}^{1 * 凶}$, Zhaolin \\ Long ${ }^{1 * \square}$ \\ 1. Department of Urology, Shunde Hospital, Southern Medical University, Guangdong, 528300, China \\ 2. Department of Urology, Nanfang Hospital, Southern Medical University, Guangzhou, 510515, China \\ 3. Institute of Biotherapy, School of Laboratory Medicine and Biotechnology, Southern Medical University, Guangzhou, 510515, China \\ 4. Zhejiang Provincial Key Laboratory of Medical Genetics, School of Life Sciences, Wenzhou Medical University, Wenzhou, 325035, China \\ *those authors contributed equally \\ $\square$ Corresponding authors: Zhaolin Long and Shihao Wu, Department of Urology, Shunde Hospital, Southern Medical University, Guangdong, 528300, China; \\ Phone: +86 0757-22318701; fax: +86 0757-22318702; Email addresses: my6q@Virginia.edu and zhangxinjimd@163.com \\ (c) Ivyspring International Publisher. This is an open access article distributed under the terms of the Creative Commons Attribution (CC BY-NC) license \\ (https://creativecommons.org/licenses/by-nc/4.0/). See http://ivyspring.com/terms for full terms and conditions.
}

Received: 2018.02.07; Accepted: 2018.08.15; Published: 2018.10.22

\begin{abstract}
Purpose: To investigate whether PD-L1 mediated adaptive resistance could occur in treatment with Anchored-GM-CSF vaccine and whether PD-1 blockade combined with Anchored-GM-CSF vaccine could induce a greater anti-tumor immune response than either immunotherapy alone.

Materials and Methods: After establishing long-established subcutaneous metastasis bladder cancer models, mice were treated with Anchored-GM-CSF vaccine and/or anti-PD-1 antibody. T-lymphocyte-cytotoxicity, flow cytometric analysis, immunohistochemical, immunofluorescence staining, $\mathrm{CD8}^{+}-\mathrm{T}$ cell apoptosis and enzyme-linked immunosorbent assays were performed to evaluate the efficacy of combination therapy with anchored-GM-CSF vaccine and PD-1 blockade and to explore the related mechanism.

Results: Anchored-GM-CSF vaccine could significantly increase the number of mature DCs and up-regulate PD-L1 expression dependent on IFN-y released from CD8 ${ }^{+} \mathrm{T}$ cells. Anchored-GM-CSF vaccine combined with anti-PD-1 antibody could effectively inhibit tumor growth and even cause regression of the established tumor. More $\mathrm{CD}^{+}$and $\mathrm{CD}^{+}{ }^{+} \mathrm{T}$ cells appeared at tumor sites and in peripheral blood in the combination therapy group than in the control groups. Splenocytes from mice of the combination therapy group exhibited the most potent cytotoxicity to MB49 cells. Apoptotic assays showed that PD-1 blockade could significantly reduce $\mathrm{CD} 8^{+} \mathrm{T}$ cells apoptosis.

Conclusions: Anchored-GM-CSF vaccines and anti-PD-1 antibodies have synergistic effects in metastatic bladder cancer treatment. PD-1 blockade can overcome immune resistance in treatment with the Anchored-GM-CSF vaccine, while Anchored-GM-CSF vaccine can enhance the efficacy of PD-1 blockade therapy.
\end{abstract}

Key words: program death ligand 1, program death receptor 1, GM-CSF, bladder cancer, vaccine

\section{Introduction}

Immunotherapy has been considered a promising approach to activating therapeutic antitumor immunity. Currently, tumor immune resistance and checkpoint blockade are under intense investigation. As reported by several studies, the most promising approaches to activating therapeutic antitumor immunity are checkpoint inhibitors and tumor vaccines ${ }^{(1)}$. 
Program death receptor-1 (PD-1) / program death ligand 1 (PD-L1) signaling plays an important role in tumor immune resistance (2). PD-L1 is expressed by tumor cells and antigen presenting cells (APCs), and PD-1 is mainly expressed on activated T cells (3). PD-L1/PD-1 interaction can lead to anergy or death of activated effector $\mathrm{T}$ cells, thereby blunting anti-tumor immune responses (4). Several clinical studies have demonstrated that PD-1/PD-L1 blockade could produce an effective clinical efficacy, but the response rate has been unsatisfactory ${ }^{(5)}$. The possible reasons for this result might be associated with a lack of tumor infiltrating lymphocytes (TILs) and PD-L1 expression in the tumor microenvironment (TME) ${ }^{(6,7) \text {. }}$

Cytokines have usually been used as adjuvants for vaccines to augment the host immune response ${ }^{(8)}$. However, inducing the desired antitumor immunity by traditional methods is difficult due to the high cytokine metabolic rate, low transgene expression, low gene transfer efficiency and biosafety concerns ${ }^{(9)}$. Thus, we recently developed a technology that rapidly and irreversibly immobilizes streptavidin (SA) tagged bioactive molecules on the surface of biotinylated MB49 bladder cancer cells (10), and proved a cytokine-modified MB49 bladder cancer cell vaccine could induce specific antitumor immunity, which effectively inhibited the growth of metastatic bladder cancer in mouse model (9). Although the granulocyte-macrophage colony-stimulating factoranchored (Anchored-GM-CSF) vaccine can induce functional $\mathrm{CD}^{+} \mathrm{T}$ cell responses in the early stage of tumor growth, and the treated mice experienced tumor regression, those tumors slowly regrew during long-term observation. The possible reason for this result might be immune evasion by the existing tumor. Tumor-infiltrating $\mathrm{CD}^{+} \mathrm{T}$ cells might gradually become dysfunctional by expressing PD-1 within the TME (11-13). In addition, PD-L1 can be expressed on the surface of cancer cells in response to IFN- $\gamma$ produced by effector $T$ cells ${ }^{(14,15)}$. This process has been termed adaptive immune resistance ${ }^{(16)}$. The PD-L1/PD-1 interaction might result in the attenuation of anti-tumor immune responses and promote tumor growth (15).

Therefore, a better approach to improve the proliferation and effector functions of $\mathrm{CD}^{+} \mathrm{T}$ cells and inhibit the growth of bladder cancer would be to combine PD-1/PD-L1 blockade with a cancer vaccine that can increase the number of tumor-specific TH1 lymphocytes. In this study, we investigated whether PD-1/PD-L1 mediated immune resistance occurred following treatment with the Anchored-GM-CSF vaccine and whether PD-1 blockade combined with the Anchored-GM-CSF vaccine could induce a strong specific antitumor immune response against bladder cancer.

\section{Materials and methods}

\section{Animals and cells}

C57BL mice (6-8 weeks) were purchased from experimental animal center of Southern Medical University (Guangzhou, China). The MB49 cell line was a carcinogen induced transitional cell carcinoma derived from C57BL/ 6 male mice. The MB49 cells were cultured in RPMI1640 containing 10\% fetal bovine serum, $1 \%$ penicillin/streptomycin in a $5 \%$ $\mathrm{CO}_{2}$ humidified incubator. MycoplasmaOUT (Genloci) was used to protect the cells against mycoplasma contamination. The MB49 cells have been authenticated and tested for mycoplasma contamination every 6-12 months. All animal studies were performed in accordance with the university guidelines of experimental animals (Ethical number: L2016045). SA-GM-CSF fusion protein and SA-GFP fusion protein were prepared by our laboratory (17).

\section{Vaccine preparation and biological activity assay}

As described in our previous study (9), MB49 bladder cancer cells in the exponential growth stage were fixed in $30 \%$ ethanol (volume/volume) for 30 minutes and then incubated with $10 \mathrm{mM}$ EZ-Link Sulfo-NHS-LC-Biotin (Pierce Biotechnology) for 1 hour at room temperature. The biotinylated MB49 cells were incubated with SA-mGM-CSF. The expression of SA-mGM-CSF on the surface of MB49 cells was assayed by flow cytometry after staining with PE-labeled anti-mouse GM-CSF monoclonal antibody. The bioactive assay of SA-GM-CSF immobilized on the surface of MB49 cells was performed according to our previously described method (10).

\section{Animal experiments}

C57BL/ 6 mice were injected in the hind leg with $1 \times 10^{6}$ MB49 cells to establish a subcutaneous tumor model. To evaluate the immunotherapy induced by Anchored-GM-CSF vaccine, mice were randomly divided into four groups (Anchored-GM-CSF, Soluble GM-CSF, Ethanol-fixed MB49 cells and PBS). Once palpable tumors developed (8 days after cell injected), Experimental group was injected subcutaneously with Anchored-GM-CSF vaccine $\left(1 \times 10^{6}\right.$ cells $/ 100 \mu$ l of PBS) every 4 days for a total of 5 doses. The other three groups received soluble SA-GM-CSF (100 $\mathrm{ng} / 100 \mu \mathrm{l}$ of PBS), ethanol-fixed MB49 cells $\left(1 \times 10^{6}\right.$ cells $/ 100 \mu \mathrm{l}$ of PBS) or PBS $(100 \mu \mathrm{l})$ every 4 days for a total of 5 doses.

To demonstrate whether PD-1 blockade can 
enhance the antitumor effect induced by the GM-anchored vaccine, mice were randomly divided into four groups (Anchored-GM-CSF+Anti-mPD-1 group, Anchored-GM-CSF+IgG, Anti-mPD-1 and IgG). For the PD-1 blockade experiments, $100 \mu \mathrm{g}$ of anti-PD-1 antibody (eBioscience, catalog number: 16-9985) or IgG (isotype control, eBioscience, catalog number: eB149/10H5) was injected intraperitoneally (i.p.) into each mouse every third day after Anchored-GM-CSF vaccine treatment. The subcutaneous tumor volume was calculated by the formula $[\pi / 6(w 1 \times w 2 \times w 2)]$, where $w 1$ represents the largest and $\mathrm{w} 2$ represents the smallest tumor diameter (10). In some experiments, $100 \mu \mathrm{g} /$ mouse of anti-IFN- $\gamma$ antibody (eBioscience, XMG1.2) was injected i.p. twice per week. Mice were sacrificed once tumors exceeded $1.5 \mathrm{~cm}^{3}$.

\section{PD-L1 upregulation by IFN-Y stimulation (in vitro)}

MB49 cells were co-cultured with IFN- $\gamma$ (25 $\mathrm{ng} / \mathrm{ml}$, Peprotech) or $\mathrm{CD}^{+} \mathrm{T}$ cells were separated from MB49 cell-bearing mice using CD8 Microbeads (Miltenyi Biotec, Auburn, CA) at a ratio of 1:1. These cells were cultured in the presence or absence of anti-IFN- $\gamma$ antibody ( $5 \mu \mathrm{g} / \mathrm{ml}$, eBioscience, XMG1.2). After 5, 10, 15 or 30 hours, the cells were stained with PE-labeled anti-mPD-L1 antibody, and PD-L1 expression was measured by flow cytometry.

\section{Flow cytometry analysis}

On day 28 after establishment of the subcutaneous tumor model, splenocytes or blood was isolated from each group. After lysing red blood cells, the remaining cells were stained with FITC-labeled anti-mCD11c (catalog number: 11-0114), PE-labeled anti-mCD80(catalog number: 12-0801), FITC-labeled anti-mCD4 (catalog number: 11-0042), or APC-labeled anti-mCD8 (catalog number: 17-0081) (all from eBioscience) for 30 minutes at room temperature. The positive cells were measured by flow cytometry.

\section{Immunohistochemistry (IHC)}

On day 28 after establishment of the subcutaneous tumor model, subcutaneous tumor tissues were collected from each group The frozen sections were then fixed with $4 \%$ paraformaldehyde and embedded in paraffin. Paraffin sections (4-5 $\mu \mathrm{m})$ were deparaffinized and rehydrated. After treatment with hydrogen peroxide, the sections were incubated with anti-mCD4 (Abcam, catalog number: EPR19514) and anti-mCD8 antibodies (eBioscience, catalog number: 14-0195) according to the instructions of the Rabbit specific HRP/DAB (ABC) Detection IHC Kit (Abcam) and restained with hematoxylin and eosin.

\section{Immunofluorescence analyses}

Tumor tissues were collected from each group. After antigen retrieval, the sections were incubated with rabbit anti-mouse PD-L1 (Abcam, catalog number: MIH6), rabbit anti-mouse PD-1 (Abcam, catalog number: RMP1-14) and /or rat anti-mouse CD8 (eBioscience, catalog number: 14-0195) antibodies. Then secondary antibodies (Alexa Fluor 647-labeled goat anti-rabbit Ab for PD-1 and PD-L1, Alexa Fluor 488-labeled goat anti-rat Ab for CD8) were applied. Slides were washed three times and mounted with a Vectashield DAPI-containing kit. The expression of PD-L1, PD-1 and CD8 in the TME was observed using a fluorescence microscope (Nikon, Eclipse E800).

\section{Tumor-specific cytotoxicity assay (CTL)}

Splenocytes were isolated from each group on day 28 after tumor injection and then stimulated by MB49 cells in the presence of human interleukin-2 (IL-2) for 5 days. These splenocytes served as effector cells and were adjusted to $1 \times 10^{5}$ cells $/ \mathrm{ml}$. MB49 cells served as target cells and were adjusted to a density of $1 \times 10^{4}$ cells $/ \mathrm{mL}$. Both cell types were added to a 96-well plate at the desired effector-to-target ratios. After incubation for 4 hours, the supernatant was collected. The lactate dehydrogenase activity was measured by the CytoTox 96 nonradioactive cytotoxicity assay.

\section{Enzyme-linked immunosorbent assay (ELISA)}

$\mathrm{CD}^{+} \mathrm{T}$ cells were isolated from the splenocytes of each group using CD8 Microbeads (Miltenyi Biotec, Auburn, CA). MB49 cells were added to CD8 ${ }^{+} \mathrm{T}$ cells at a ratio of 5:1 and incubated for 24 hours at $37^{\circ} \mathrm{C}$. The concentration of IFN- $\gamma$ was measured by ELISA (eBioscience).

\section{Apoptotic assay}

$\mathrm{CD}^{+} \mathrm{T}$ cells were isolated from splenocytes of the Anchored-GM-CSF vaccine group (on day 28 after tumor cell injection) using immunomagnetic microbeads. CD8 ${ }^{+}$T cells were cultured with MB49 cells on 24-well plates and treated with anti-PD-1 or IgG antibody for 72 hours. Then, cell apoptosis was measured by flow cytometry using an annexin V/FITC and propidium iodide (PI) apoptosis detection kit (BD Bioscience). Annexin V(+)/PI(-) and annexin $\mathrm{V}(+) / \mathrm{PI}(+)$ indicated apoptosis and necrosis, respectively.

\section{Statistical analysis}

All experimental group values were analyzed from representative experiments. The differences in tumor volume between groups were compared using 
repeated measures design. Statistical analyses of flow cytometry, ELISA, IHC and Immunofluorescence data were determined by one-way ANOVA. Statistical analysis was performed using SPSS (version 19.0). $P<$ 0.05 was considered as indicative of statistical significance.

\section{Results}

\section{Immobilization and biological activity of SA-GM-CSF}

Flow cytometric analysis showed that SA-GMCSF could be efficiently anchored on the surface of MB49 cells (Figure 1a). The bioactivity of SA-GM-CSF immobilized on the surface of MB49 cells was assessed by bone marrow cell proliferation, with SA-GFP as a negative control. The results demonstrated that SA-mGM-CSF anchored on the surface of MB49 cells retained bioactivity in a dose dependent manner (Figure 1b). a

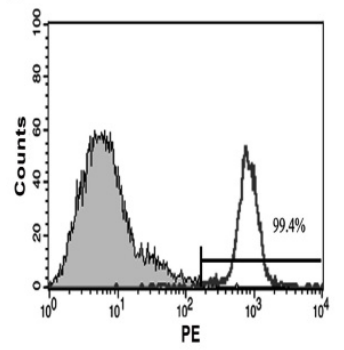

b

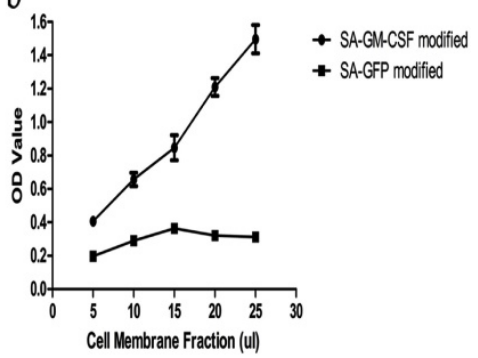

Figure 1. Immobilization and biological activity of SA-mGM-CSF on the surface of MB49 cells. a. Results of the flow cytometry analysis showed that SA-mGM-CSF could be efficiently anchored on the surface of MB49 cells, with unbiotinylated MB49 cells as a negative control. $\mathbf{b}$. The biological activity of GM-CSF on the surface of MB49 cells was assessed, with SA-GFP as a negative control. OD: optical density.

\section{Anchored-GM-CSF vaccine induced DC activation, but failed to induce regression of the established tumor}

To assess the effect of Anchored-GM-CSF vaccine on DC maturation, mature DCs $\left(\mathrm{CD} 11 \mathrm{c}^{+}\right.$ $\mathrm{CD} 80^{+}$) in the spleens of mice were measured by flow cytometry. The results showed that the AnchoredGM-CSF vaccine could significantly increase the number of mature DCs population (Figure 2a,b).

According to the results, treatment with the Anchored-GM-CSF vaccine alone modestly slowed tumor growth but failed to induce the regression of established tumors (Figure 2c).

\section{Anchored-GM-CSF vaccine treatment increased PD-LI expression depending on IFNy}

First, $\mathrm{CD} 8{ }^{+} \mathrm{T}$ cells were isolated from the spleens of untreated $\mathrm{C} 57 \mathrm{BL} / 6$ mice, the isolation rate was 98.5\% (Figure 3a). To confirm the role of IFN-y in the regulation of PD-L1 expression in tumor cells (16), MB49 cells were cultured with $\mathrm{CD}^{+} \mathrm{T}$ cells or IFN- $\gamma$ for 72 hours. The results showed that both $\mathrm{CD}^{+} \mathrm{T}$ cells and IFN $\gamma$ could up-regulate PD-L1 expression on MB49 cells, and blocking IFNY could inhibit the upregulation of PD-L1 expression (Figure 3b). In vivo study, we isolated $\mathrm{CD}^{+} \mathrm{T}$ cells and noted an increased level of IFNY secretion from $\mathrm{CD}^{+} \mathrm{T}$ cells in Anchored-GM-CSF vaccine treatment group by ELISA (Figure 3c). We also found that AnchoredGM-CSF vaccine could upregulate the expression of PD-L1 within the TME, but the upregulated expression of PD-L1 could be significantly diminished when treated with IFN $\gamma$ neutralizing antibody (Figure $3 \mathrm{~d}, \mathrm{e})$. Thus, upregulation of PD-L1 was triggered in an IFN $\gamma$-dependent mechanism.

\section{PD-1 blockade enhanced the therapeutic efficacy of the Anchored-GM-CSF vaccine}

Combination treatment with the Anchored-GMCSF vaccine and PD-1 blockade produced a better anti-tumor response in vivo, significantly delaying tumor growth $(P<0.05)$ (Figure 4a). Meanwhile, anti-IFNY antibody abrogated the anti-tumor response induced by Anchored-GM-CSF vaccine or anti-PD-1, which was consistent with the adaptive immune resistance mechanism (Figure 4a).

To further evaluate the effect of the combined treatment on different $\mathrm{T}$-cell subsets, $\mathrm{CD} 4^{+}$and $\mathrm{CD} 8^{+} \mathrm{T}$ lymphocytes in peripheral blood and the TME were detected by flow cytometry or IHC. The results showed that $\mathrm{CD}^{+}$and $\mathrm{CD}^{+} \mathrm{T}$ cells in the Anchored-GM-CSF vaccine combined with anti-PD-1 group were notably increased compared to those in the other groups (Figure $4 b, c, e, f)$.

To investigate the specific antitumor immunity induced by combination therapy, splenocytes served as effector cells and MB49 cells served as target cells. The percent cytotoxicity of tumor-specific cytotoxic $\mathrm{T}$ lymphocytes in each group was determined by the lactate dehydrogenase activity level. (17) The results of the CTL assay showed that PD-1 blockade could significantly enhance the tumor-specific cytotoxic activities of the Anchored-GM-CSF vaccine relative to those of the Anchored-GM-CSF vaccine monotherapy (Figure 4d).

\section{PD-1 blockade enhanced the antitumor efficacy of Anchored-GM-CSF therapy by reducing apoptosis of}

PD-1/PD-L1 signaling might promote $\mathrm{T}$ cells apoptosis (18). To define PD-1 expression on the surface of $\mathrm{CD}^{+} \mathrm{T}$ cells in the TME after combined 
treatment, tumor tissues were collected from each group. The results of the immunofluorescence assay showed that the number of $\mathrm{PD}-\mathrm{1}^{+} \mathrm{CD} 8^{+} \mathrm{T}$ cells was significantly increased after combined treatment (Figure 5a,b). These findings revealed that PD-1 blockade could enhance the therapeutic efficacy of the
Anchored-GM-CSF vaccine. To explore the mechanism, we further investigated the effect of combined treatment on $\mathrm{CD}^{+} \mathrm{T}$ cell apoptosis and showed that PD-1 blockade could significantly decrease $\mathrm{CD}^{+} \mathrm{T}$ cell apoptosis (Figure $5 \mathrm{c}, \mathrm{d}$ ).

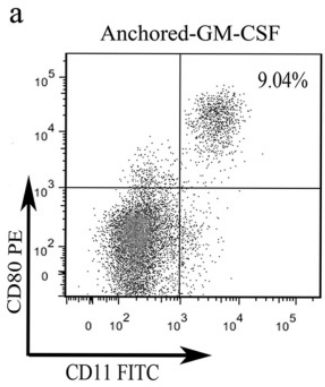

b

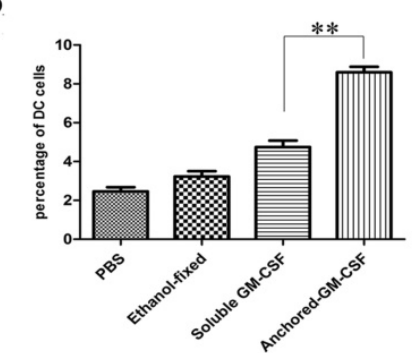

Soluble GM-CSF

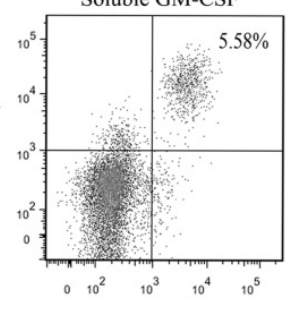

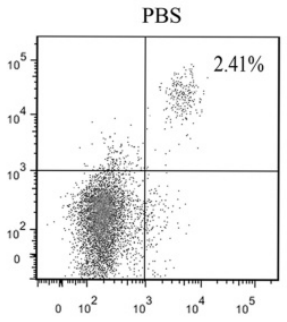

c

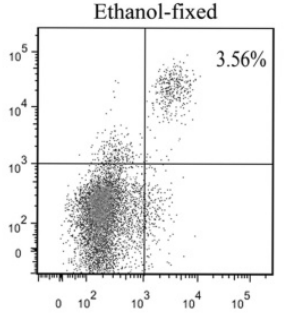

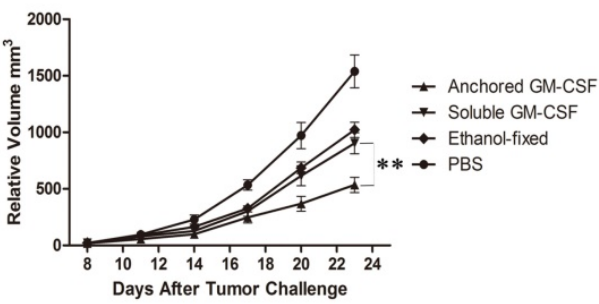

Figure 2. Anchored-GM-CSF vaccine increased DC activation and induced an anti-tumor response. a and b. After Anchored-GM-CSF vaccine treatment, splenocytes were isolated from each group and stained with PE-labeled anti-mCD 1 lc and FITC-labeled anti-mCD80 antibodies. The CD $11 c^{+} C D 80+D C s$ were considered mature dendritic cells and were measured by flow cytometry. c. The tumor volume was recorded. The anchored-GM-CSF vaccine could effectively reduce tumor growth compared with control treatment. All experiments were replicated at least 3 times. $* * p<0.01$.

a

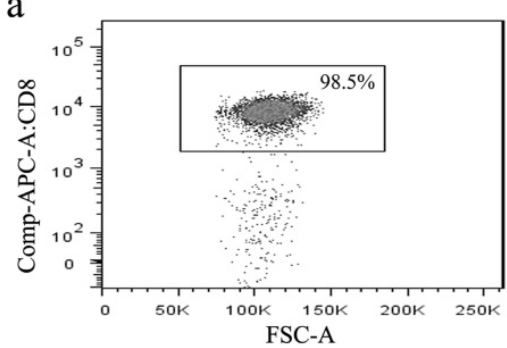

b

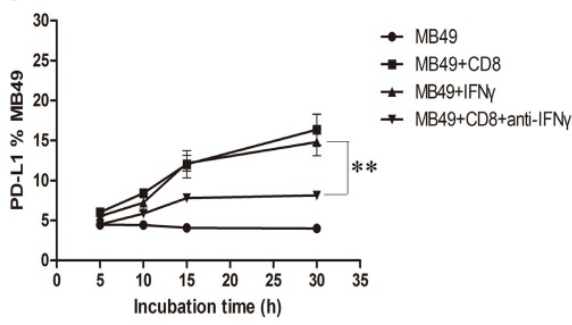

$\mathrm{C}$

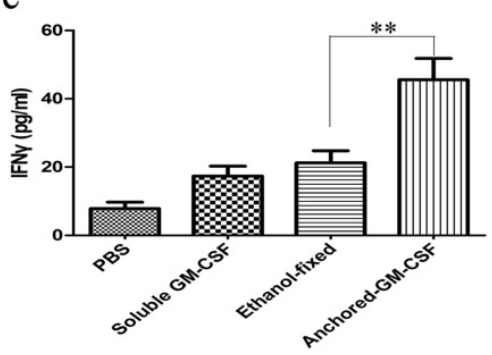

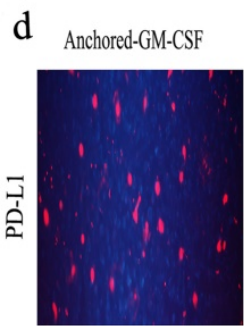
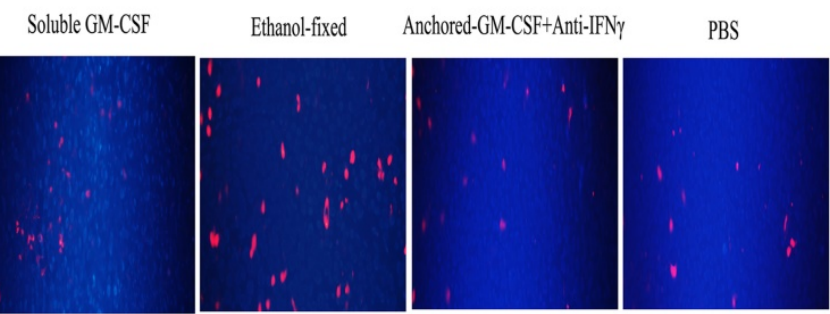

$\mathrm{e}$

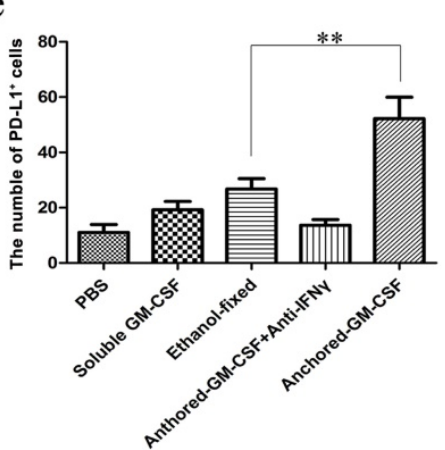

Figure 3. Anchored-GM-CSF vaccine up-regulated PD-L1 expression depending on IFNy. a. After Anchored-GM-CSF vaccine treatment, CD8 ${ }^{+}$T cells were isolated from the spleens of untreated C57BL/6 mice by CD8a (Ly-2) MicroBeads and CD8+ $T$ cells were detected by flow cytometry. $\mathbf{b}$. CD8 ${ }^{+} T$ cells could upregulate PD-L1 expression on MB49 cells in an IFNy -dependent manner in vitro. c. CD8 ${ }^{+} \mathrm{T}$ cells were collected from each group, and the concentrations of serum IFNY in each group were measured by ELISA. $\mathbf{d}$ and e. Tumor tissues from mice treated with Anchored-GM-CSF vaccine, Soluble GM-CSF, Ethanol-fixed, PBS, or Anchored-GM-CSF vaccine administered with neutralizing IFNy antibody were harvested and stained with anti-mPD-LI antibodies. Then, a secondary antibody was used to assess PD-LI positive cells. Blinded quantitation was performed in 10 different fields at 40x magnification. All experiments were replicated at least 3 times. $* * p<0.01$. 

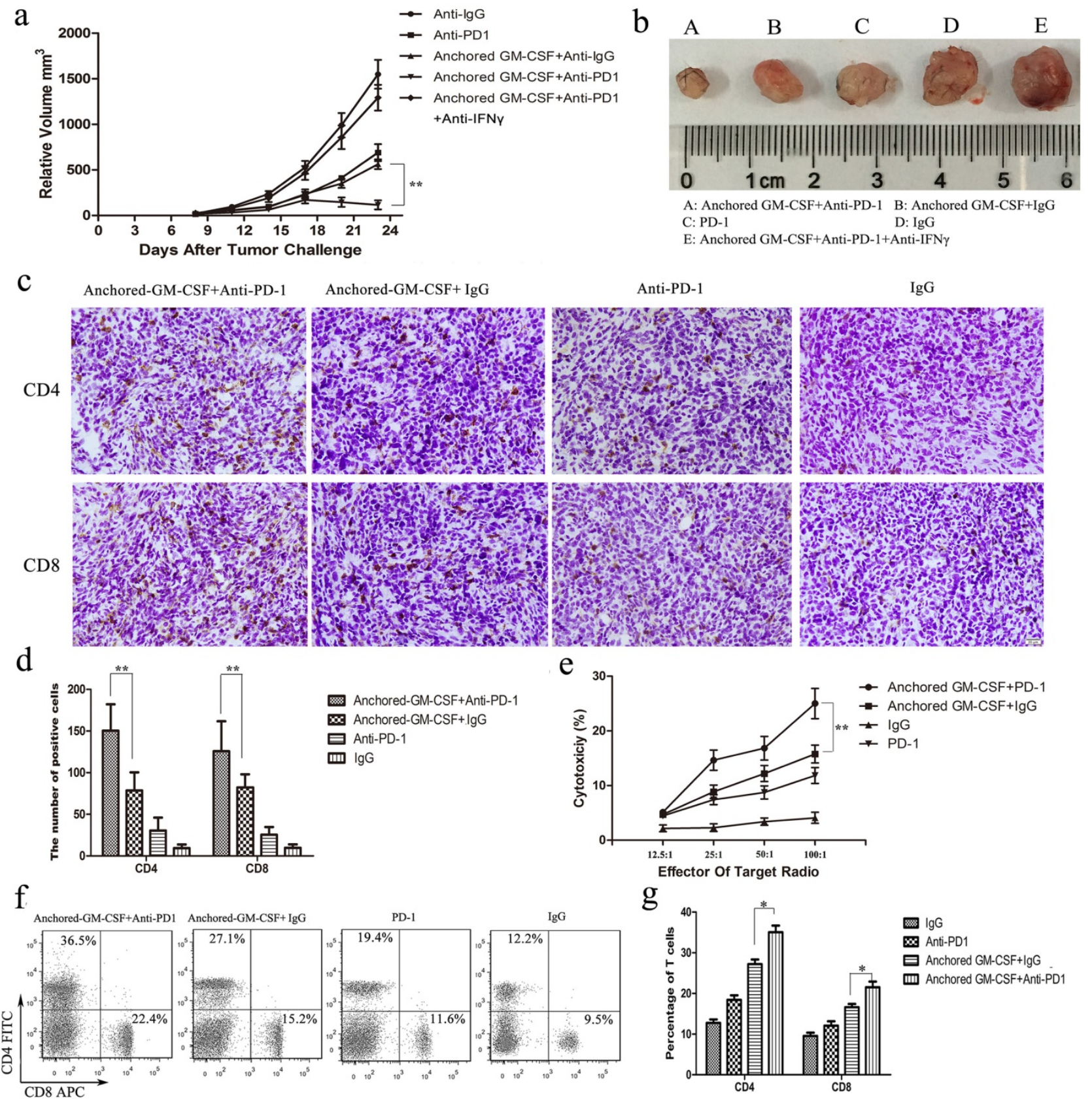

Anti-PD-1
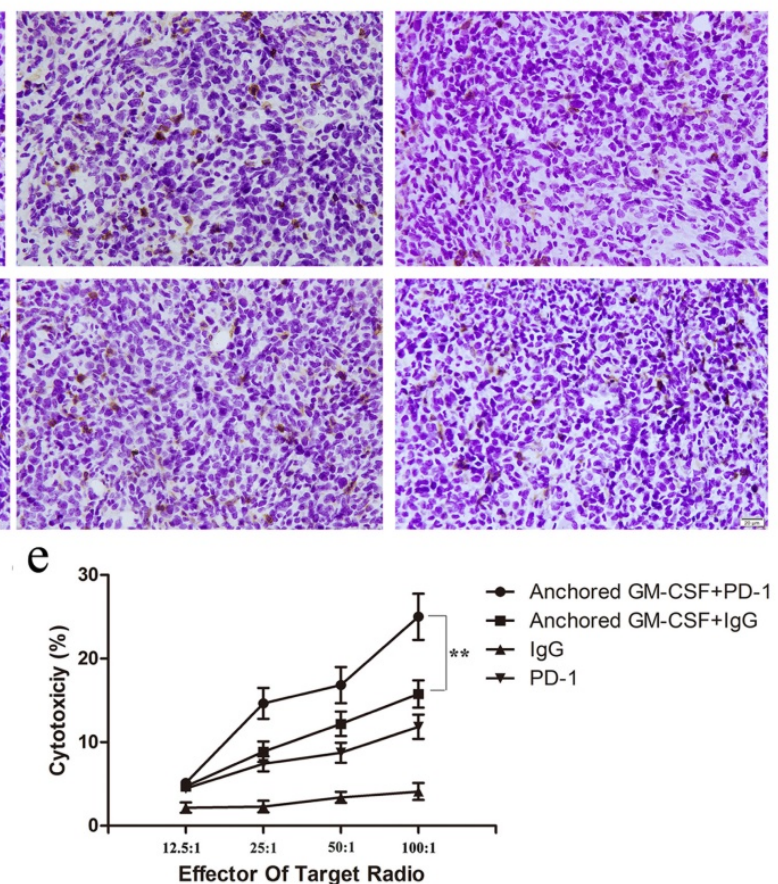

$\mathrm{g}$

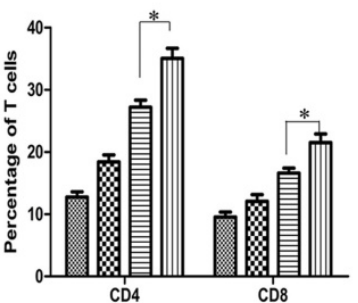

IgG

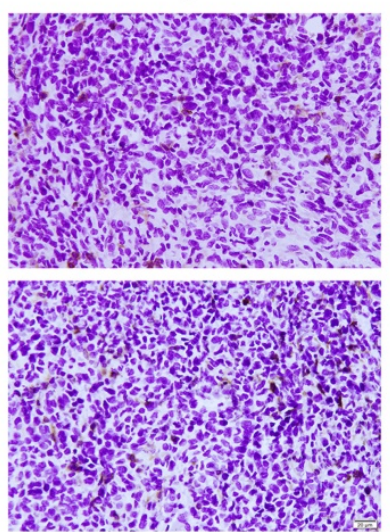

Figure 4. Anchored-GM-CSF vaccine combined with PD-1 blockade enhanced the T lymphocyteresponse and reversed adaptive immune resistance. a. The tumor volume in each treated group was recorded after tumor challenge. Anchored-GM-CSF vaccine treatment combined with anti-PD-1 could effectively inhibit tumor growth compared to Anchored-GM-CSF vaccine or anti-PD-1 monotherapy alone. Anti-IFNy antibody abrogated tumor regression. b. Gross image of the tumor in each group. $\mathbf{c}, \mathbf{d}, \mathbf{f}$ and $\mathbf{g} . \mathrm{CD} 4^{+}$and $\mathrm{CD} 8^{+} \mathrm{T}$ lymphocytes in blood and the TME were measured by flow cytometry and IHC. d. Splenocytes were isolated from each group and stimulated by hIL-2. MB49 cells served as target cells. Supernatants were collected for the nonradioactive cytotoxicity assay. All experiments were replicated 3 times. $* p<0.05, * * p<0.01$.

\section{Discussion}

In this study, we demonstrate that combination therapy with PD-1 blockade and Anchored-GM-CSF vaccine could synergistically induce anti-tumor immune responses and significantly promote tumor regression. We found that treatment with Anchored-GM-CSF alone could promote DC activation and enhance the infiltration of effector T-cells into tumor tissue, but could not completely inhibit tumor growth. The tumor microenvironment contains a strong immunosuppressive network. PD-1/PD-L1 signaling is an important immune evasion mechanism of the established tumor. The results of further studies showed that PD-L1 expression in the TME and PD- 1 expression on CD8+ $T$ cells were significantly upregulated after AnchoredGM-CSF vaccine treatment. In this study, we tested whether it is possible to optimize our vaccination platform by blocking the PD-1/PD-L1 axis. 
PD-1/PD-L1 signaling may mediate immune resistance by promoting $\mathrm{CD} 8^{+} \mathrm{T}$ cells apoptosis ${ }^{(18)}$. In the early stage of tumor growth, immunogenic cancer cells can induce functional CD8 ${ }^{+} \mathrm{T}$ cell responses ${ }^{(19)}$. With tumor growth, the establishment of a tumor microenvironment in which cancer cells are embedded in a suppressive tumor stroma, greatly impaires antitumor immunity (20). The proliferation and functions of $\mathrm{CD}^{+} \mathrm{T}$ cells are usually impaired by PD-1/PD-L1 interactions (15). Because of the existence of this immune resistance mechanism, we administered a combination treatment with anti-PD-1 antibody and the Anchored-GM-CSF vaccine. The results showed that tumor growth could be effectively inhibited. The combination therapy improved the proliferation and function of $\mathrm{CD}^{+} \mathrm{T}$ cells, and $\mathrm{CD} 8^{+} \mathrm{T}$ cells apoptosis was also decreased (Figure 4 and Figure 5c,d).

At present, the adaptive immune resistance mechanism is being investigated. IFN- $\gamma$ is a known mediator of PD-L1 expression in cancer cell lines. It is intriguing to explore whether similar mechanisms underlie tolerance to vaccine-based cancer immunotherapy. In the in vivo and in vitro studies, we found that PD-L1 expression was upregulated by IFNY produced by $\mathrm{CD}^{+} \mathrm{T}$ cells. Anti-IFNY antibody significantly inhibited PD-L1 expression on MB49 cells in vitro and abrogated the regression of tumors in vivo (Figure 3 and Figure 4a).

PD-1/PD-L1 blockade monotherapy has already been successfully administrated in various cancers and has reinvigorated interest in the treatment of metastatic bladder cancer (21). Several clinical studies have demonstrated that PD-1/PD-L1 blockade could produce a potent anti-tumor effect $(22,23)$. However, the response rates have been unsatisfactory (approximately 20\%) (5). A possible reason for this result might be the lack of TILs. In addition, MB49 tumor cells express PD-L1 (Figure 3d,e), which increases with tumor growth ${ }^{(20)}$. Therefore, anti-PD-1 monotherapy did not effectively inhibit tumor growth. An effective immunotherapy should combine checkpoint blockade with a driver of tumor-specific immunity. The Anchored-GM-CSF vaccine can increase tumor-specific IFN- $\gamma$-secreting TH1 lymphocytes. Therefore, PD-1 blockade combined with Anchored-GM-CSF vaccine could effectively inhibit the tumor growth and improve the tumor regression rate (Figure 4).

Overall, this study demonstrates that AnchoredGM-CSF vaccine and PD-1 blockade have synergistic effects in the treatment of bladder cancer. PD-1 blockade can overcome immune resistance during treatment with Anchored-GM-CSF vaccine, while Anchored-GM-CSF vaccine can enhance the efficacy of PD-1 blockade therapy. Based on our preclinical evidence, a clinical trial is currently ongoing to evaluate the efficacy of the Anchored-GM-CSF vaccine against human bladder cancer. This study may also provide a preclinical basis for applying the combination therapy with Anchored-GM-CSF vaccine and PD-1 blockade in human bladder cancer.
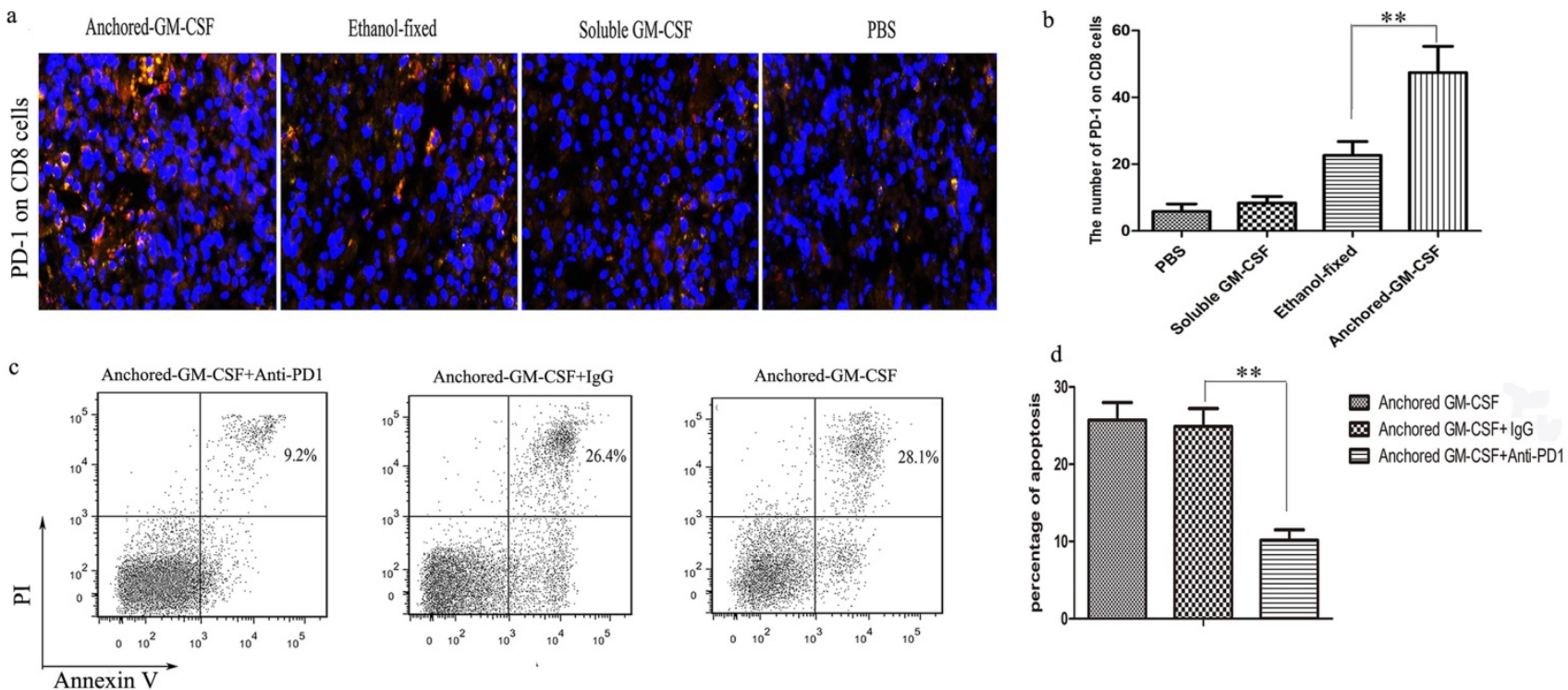

Figure 5. PD-1 blockade reduced CD8+ ${ }^{+}$-cells apoptosis. a and b. PD-1 expressed in the TME was measured by immunofluorescence. CD $8^{+}$T-cells were detected using AlexaFluor 488 staining via the green channel and PD-1+ cells were detected using AlexaFluor 647 staining via the red channel. Yellow fluorescence indicates PD-1+CD8 + T-cells. $\mathbf{c}$ and d. CD8 ${ }^{+} \mathrm{T}$ cells were separated from the tumor tissues of mice in Anchored-GM-CSF vaccine group on day 28 after the model was established. These T cells were cultured with MB49 cells on 24-well plates and treated with anti-PD-1 or lgG antibody for 72 hours. Then the cells were subjected to an annexin- $\mathrm{V}$ and $\mathrm{PI}$ binding assay. The numbers in the graph indicate the percentage of Annexin $\mathrm{V}(+) / \mathrm{PI}(-)$ and $\mathrm{Annexin} \mathrm{V}(+) / \mathrm{PI}(+)$ cells. $\mathrm{All}$ experiments were replicated 3 times. ${ }^{* *} p<0.01$ 


\section{Abbreviations}

SA: streptavidin; GM-CSF: granulocyte-macrophage colony-stimulating factor; CTL: cytotoxicity of T lymphocyte; DCs: dendritic cells; PD-1: program death receptor-1; PD-L1: program death ligand 1; IHC: immunohistochemistry; ELISA: enzyme-linked immunosorbent assay; TME: tumor micro environment.

\section{Acknowledgments}

Dr. I. C. Summerhayes, Lahey Clinic, Burlington, Massachusetts, provided MB49 cells.

\section{Funding}

This work was supported by grants from the Natural Science Foundation of China (No.81373122).

\section{Authors' Contributions}

Experimental design: Zhaolin Long, Shihao $\mathrm{Wu}$ and Xinji Zhang. Performed the experiments: Xinji Zhang, Xiaojun Shi, Lijun Mo and Jinlong Li. Data analysis and interpretation: Lijun Mo and Zhiming Hu. Drafted the manuscript and figures: Jimin Gao and Zhiming Hu. Manuscript revision: Zhaolin Long, Xinji Zhang and Jinlong Li. All the authors approved the final manuscript.

\section{Competing Interests}

The authors have declared that no competing interest exists.

\section{References}

1. Rekoske BT, Olson BM, McNeel DG. Antitumor vaccination of prostate cancer patients elicits PD-1/PD-L1 regulated antigen-specific immune responses[J]. Oncoimmunology, 2016. 5(6):e1165377.

2. Drake CG, Lipson EJ, Brahmer JR. Breathing new life into immunotherapy: review of melanoma, lung and kidney cancer[J]. Nat Rev, 2014. 11(1): 24-37.

3. Francisco LM, Sage PT, Sharpe AH. The PD-1 pathway in tolerance and autoimmunity[J]. Immunol Rev, 2010. 236:219-42.

4. Dong H, Strome SE, Salomao DR, et al. Tumor-associated B7-H1 promotes T-cell apoptosis: a potential mechanism of immune evasion[J]. Nat Med, 2002. 8:793-800.

5. Bellmunt J, Powles T, Vogelzang NJ. A review on the evolution of PD-1/PD-L1 immunotherapy for bladder cancer: The future is now[J]. Cancer Treat Rev, 2017. 54:58-67.

6. Blank C, Brown I, Peterson AC, et al. PD-L1/B7H-1 inhibits the effector phase of tumor rejection by $\mathrm{T}$ cell receptor (TCR) transgenic CD8 ${ }^{+} \mathrm{T}$ cells[J]. Cancer Res, 2004. 64(3):1140-5.

7. Soares KC, Rucki AA, Wu AA, Olino K, Xiao Q, Chai Y, et al. PD-1/PD-L1 blockade together with vaccine therapy facilitates effector $\mathrm{T}$ cell infiltration into pancreatic tumors. J Immunother 2015; 38(1):1-11.

8. Singh NP, Yolcu ES, Taylor DD, Gercel-Taylor C, Metzinger DS, Dreisbach SK, et al. A novel approach to cancer immunotherapy: tumor cells decorated with CD80 generate effective antitumor immunity. Cancer Res. 2003;63:4067-4073.

9. Zhang X, Shi X, Li J, Hu Z, Zhou D, Gao J, et al. A novel therapeutic vaccine of mouse GM-CSF surface modified MB49 cells against metastatic bladder cancer. J Urol. 2012 Mar;187(3):1071-9.

10. Hu Z, Tan $\mathrm{W}$, Zhang L, Liang Z, Xu C, Su H, et al. A novel immunotherapy for superficial bladder cancer by intravesical immobilization of GM-CSF. J Cell Mol Med 2010;14: 1836-44

11. Thompson RH, Dong H, Lohse CM, Leibovich BC, Blute ML, Cheville JC, et al. PD-1 is expressed by tumor-infiltrating immune cells and is associated with poor outcome for patients with renal cell carcinoma. Clin Cancer Res. 2007; 13:1757-1761.
12. Yamamoto R, Nishikori M, Kitawaki T, Sakai T, Hishizawa M, Tashima M, et al. PD-1-PD-1 ligand interaction contributes to immunosuppressive microenvironment of Hodgkin lymphoma. Blood. 2008; 111:3220-3224.

13. Ahmadzadeh M, Johnson LA, Heemskerk B, Wunderlich JR, Dudley ME, White DE, et al. Tumor antigen-specific CD8 T cells infiltrating the tumor express high levels of PD-1 and are functionally impaired. Blood. 2009; 114:1537-1544.

14. Spranger S, Spaapen RM, Zha Y, Williams J, Meng Y,Ha TT, et al. Up-regulation of PD-L1, IDO, and T(regs) in the melanoma tumor microenvironment is driven by CD8(+) T cells. Sci Transl Med. 2013; 5:200ra116.

15. Taube JM, Anders RA, Young GD, Xu H, Sharma R, McMiller TL, et al. Colocalization of inflammatory response with B7-h1 expression in human melanocytic lesions supports an adaptive resistance mechanism of immune escape. Sci Transl Med. 2012 Mar 28;4(127):127ra37.

16. Pardoll DM. The blockade of immune checkpoints in cancer immunotherapy. Nature Reviews Cancer. 2012; 12:252-264.

17. Zhang X, Shi X, Li J, Hu Z, Guo F, Huang X, et al. Novel Immunotherapy for Metastatic Bladder Cancer Using Vaccine of Human Interleukin-2 Surface-modified MB49 Cells. Urology. 2011 Sep;78(3):722.e1-722.e6.

18. Barber DL, Wherry EJ, Masopust D, Zhu B, Allison JP, Sharpe AH, et al. Restoring function in exhausted CD8 T cells during chronic viral infection. Nature. 2006; 439:682-687.

19. Kline J, Brown IE, Zha YY, Blank C, Strickler J, Wouters H, et al. Homeostatic proliferation plus regulatory T-cell depletion promotes potent rejection of B16 melanoma. Clin Cancer Res. 2008;14:3156-3167.

20. Binder DC, Engels B, Arina A, Yu P, Slauch JM, Fu YX, et al. Antigen-specific bacterial vaccine combined with anti-PD-L1 rescues dysfunctional endogenous T cells to reject long-established cancer. Cancer Immunol Res. 2013 Aug;1(2):123-33.

21. Zhou TC, Sankin AI, Porcelli SA, Perlin DS, Schoenberg MP, Zang X. A review of the PD-1/PD-L1 checkpoint in bladder cancer: From mediator of immune escape to target for treatment. Urol Oncol. 2017 Jan;35(1):14-20.

22. Powles T, Eder JP, Fine GD, Braiteh FS, Loriot $Y$, Cruz C et al. MPDL3280A (anti-PD-L1) treatment leads to clinical activity in metastatic bladder cancer. Nature. 2014 Nov 27;515(7528):558-62.

23. Rosenberg JE, Hoffman-Censits J, Powles T, van der Heijden MS, Balar AV, Necchi A, et al. Atezolizumab in patients with locally advanced and metastatic urothelial carcinoma who have progressed following treatment with platinum-based chemotherapy: a single-arm, multicentre, phase 2 trial. Lancet. 2016 May 7;387(10031):1909-20. 